\title{
Fabrication of PEG Thin Films by Irradiation of Atmospheric Pressure Glow Discharge on the Polyethylene Powder
}

\author{
Masuhiro Kogoma, Kenji Tuji , and Kunihito Tanaka \\ Department of Chemistry, Faculty of Science and Technology, Sophia University, Tokyo, Japan \\ 7-1 Kioicho, Chiyoda-ku, Tokyo, Japan
}

\begin{abstract}
Water insoluble polyethylene glycol (PEG ) films as a core-shell of polymer powder were fabricated by the combination techniques of adsorption of PEG on the powder surfaces and atmospheric glow discharge(APGD) irradiation to the surface to take cross linking reaction of the adsorbed PEG. PEG 2000 was used as the precursor of the core-shell films. The obtained films on the powder surfaces were investigated by XPS,ATR-FTIR and wettability measurement using the capillary raise method.
\end{abstract}

Keywords:PEG, APGD, Polyethylene powder

\section{Introduction}

Polyethylene glycol(PEG or PEO) has a non-fouling property by inhibition of protein or cell adsorption. However, to obtain the bio-compatibility surface, strong chemical bonds between the substance and PEG is required, because PEG is soluble in water or some organic solvent.

Few kind of process to fix the PEG was developed in the plasma processing technology. A method of grafting of PEG on to deposited amorphous silica film obtained by plasma CVD was developed by Alcantara et.al[1]. However, the grafting of PEG needs long time to achieve the desirable thick film. From the point of view, the method of CASING(cross-linking by activated species of inert gas) was adopted to take the cross-link reaction of physically coated low molecular PEG to have a thicker film in one time [2]. The cross-liked PEG films on the LDPE films by irradiation of Ar plasma in low pressure show a large increase of the hydrophilicity and reduction of baboon fibrinogen adsorption.

We previously reported the surface treatment in the atmospheric pressure glow discharge plasma[3]. Atmospheric discharge is much lower in cost for industry than that obtained in low pressure plasma.

PEG film has not only anti-fouling of protein but also hydrophilicity. From the reason, we can apply the PEG film for some enzyme reaction as a carrier layer with out of any protein adsorption on the surface. However, the catalytic chemical reaction needs much larger surface area than that of the film. Moreover, polyethylene fine powder surface oxidation treatment using APGD was reported by our group[4]. If we obtain the PEG film on the fine powder surface, we can enlarge the surface area than that of the plane film to improve the catalytic process. We will report the combination study of the both processes to attain the PEG fixation on the fine polyethylene powder surface by using raw PEG adsorption and irradiation of APGD.

\section{Experimental}

Scheme 1. Polyethylene powder oxidation in APGD.

Before the PEG adsorption, polyethylene powder surfaces should be oxidized because polyethylene is one of the most difficult materials to absorb hydrophilic material on the surfaces. So the non oxidized polyethylene powder will not absorb PEG because the hydrophilic material. The powder is oxidized by APGD in advance of PEG absorption on the powder to refine the surface wettability of PE.

$\mathrm{He}(3 \mathrm{slm})$ gas is mixed with $50 \mathrm{sccm}$ of $\mathrm{O}_{2}$, then introduced in to the discharge zone with PE powder which is introduced from powder pool and $1.8-2.5 \mathrm{~kW}$ of $\mathrm{RF}(13.56 \mathrm{MHz})$ high voltage is applied to have APG discharge.

$$
\begin{array}{lll}
\text { Received } & \text { April } & \text { 2, } 2010 \\
\text { Accepted } & \text { May } & \text { 7, } 2010
\end{array}
$$


Scheme 2. PEG adsorption on PE powders

$1-10 \%$ of PEG $2000 \mathrm{MW}$ is dissolved in ethanol(Kantou kagaku Co,LTD). The oxidized polyethylene powder $(8 \mu \mathrm{m} \phi$, high density PE) is charged in to the solution and kept during 30min to two hours in room temperature to absorb PEG. PEG -PE powder is separated with filter then dried. The dried powder will be irradiated in APGD to take cross linking reaction of the adsorbed PEG .

Scheme 3. Cross-linking reaction using APGD Figure 1 shows the schematics of the plasma system to treat the powder by CASING reaction in the atmospheric pressure. Some weighted PEG absorbed PE powder is introduced from the powder pool to the plasma reaction zone using He flow. We used RF (13.56 MHz) discharge to treat the powder.

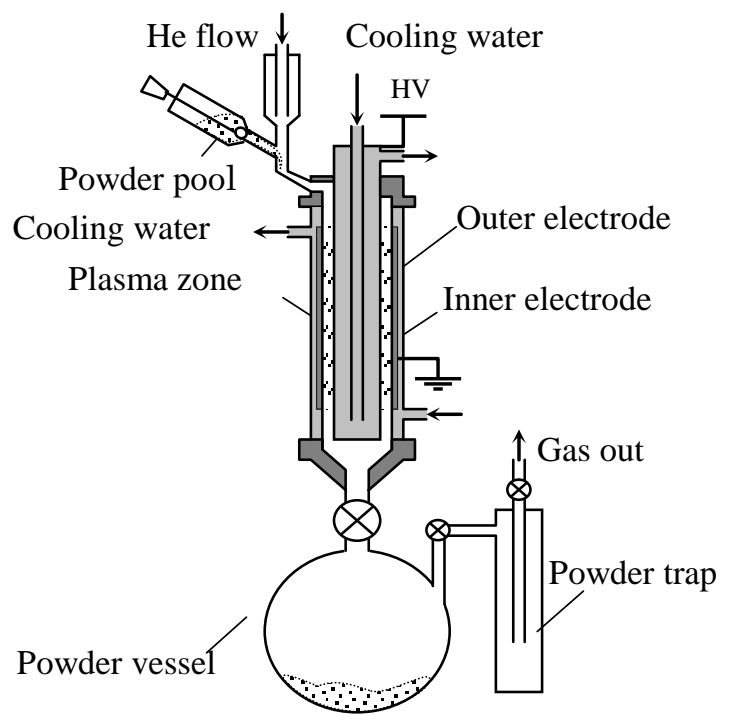

Fig.1 Shematic diagram of the reactor used in this study.

The RF power is modulated by $10 \mathrm{kHz}$ pulse of $50 \%$ on time and $50 \%$ of off time to decrease the gas temperature in the plasma zone. The plasma activated particles passing through the $\mathrm{He}$ discharge are collected in the vessel.

Table 1. Discharge conditions for $5 \mathrm{~g}$ of sample powder

\begin{tabular}{lr}
\hline Discharge Frequency / MHz & 13.56 \\
Discharge Power / kW & $1.8-2.5$ \\
Discharge time / min & 30 \\
He Flow Rate / slm & 3 \\
\hline
\end{tabular}




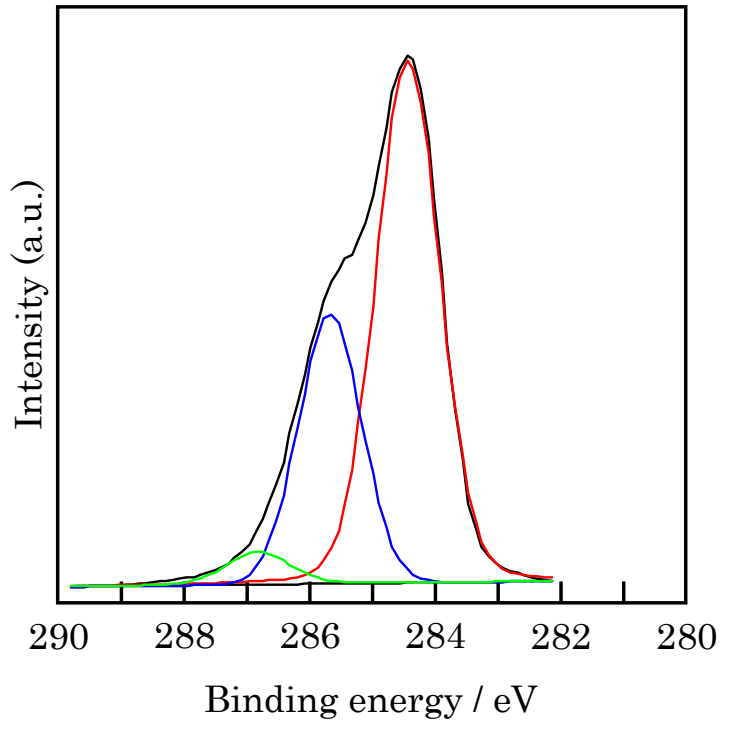

Fig. 3 XPS $\mathrm{C}_{1 \mathrm{~s}}$ spectra of $10 \%$ PEG absorbed PE powder.

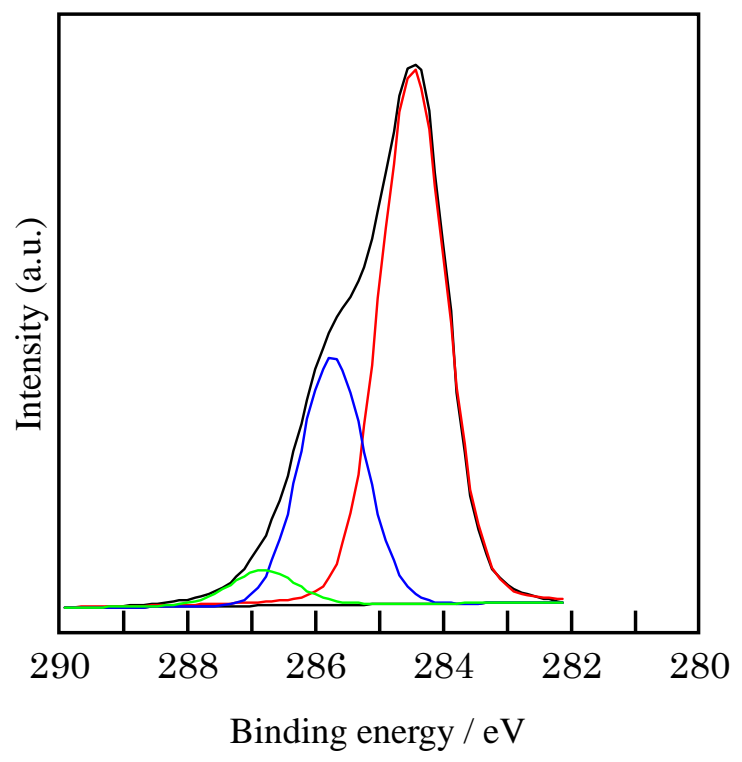

Fig. 4 XPS $\mathrm{C}_{1 \mathrm{~s}}$ spectra of absorbed PE powder irradiated by APGD in the discharge apparatus

Figure 4 shows the XPS $\mathrm{C}_{1 \mathrm{~s}}$ spectra of $10 \% \mathrm{ab}$ sorbed PEG. The height of - $\mathrm{CO}$ bonds is slightly decreased in figure 4 than that of in figure 3 . We suppose that a part of - $\mathrm{CO}$ bonds of the surfaces were dissociated by charged of meta stable exited He atom.

Figure 5 shows the ATR-FTIR spectra of untreated and treated PE surfaces. Oxide $\mathrm{PE}(\mathrm{b})$ shows some increasing of $\mathrm{C}=\mathrm{O}$ stretching peak at
$1704 \mathrm{~cm}^{-1}$, but if adsorbed by PEG in (c), strong $\mathrm{C}-\mathrm{O}-\mathrm{C}$ stretching peak at $1114 \mathrm{~cm}^{-1}$ will be appeared attributed to the chemical structure of raw PEG. When the plasma irradiation takes place on the surface of (c), $\mathrm{CH}$ stretching peaks at $2915 \mathrm{~cm}^{-1}$ and $2850 \mathrm{~cm}^{-1}$ in (d) are modified and deformation by cross linking structure on the PEG film surfaces.

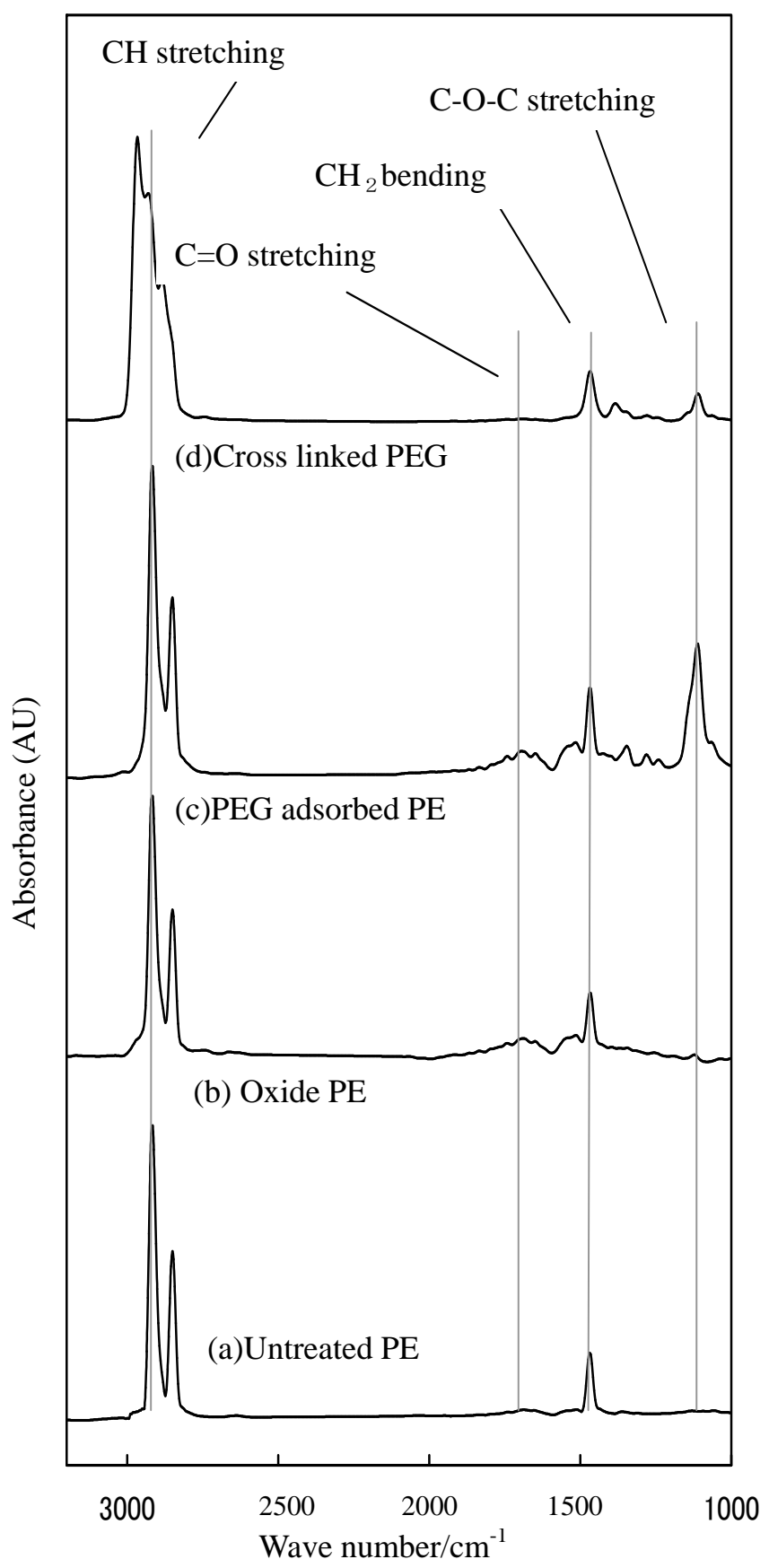

Fig.5. ATR-FTIR spectra of PE surfaces of (a)Untreated PE, (b)Oxide PE,(c)PEG adsorbed PE and (d)Cross linked PEG PE. 
Finally we measured the surface wettability of the treated and untreated powders using with capillary raise method as shown in bellow. Surface wettability is normally measured by contact angle measurement of liquid droplet on the flat surface. But the method is not usable for the particle surface.

Figure 6 shows the capillary raise method for the surface wettability measurement of the powder.

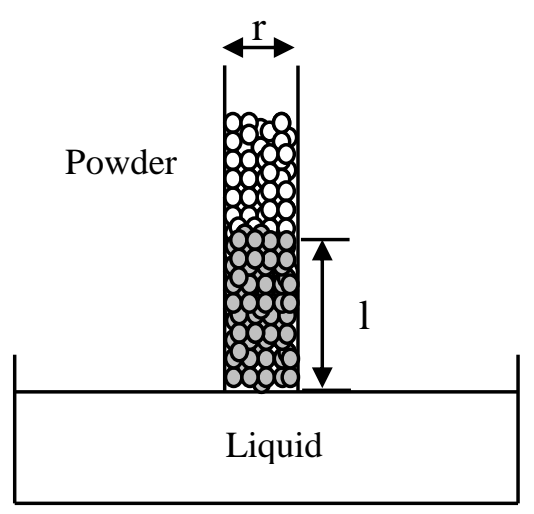

Fig.6 Schematics of Capillary raise method

The powder is stuffed in the tube then the bottom is stopped by the filter paper and soaked in the test liquid. The liquid is raising path through the clink of the powder particles then the raising height is measured with soaking time. In the method, the raising rate $(\mathrm{dl} / \mathrm{dt})$ of the liquid is shown by the equation (1). In the equation, $r$ is the inside radius of the tube $(6 \mathrm{~mm} \phi), \eta$ is the liquid viscosity, $\gamma_{1}$ is the liquid surface tension, $\theta$ is the contact angle of the surface, $\Delta \mathrm{p}$ is the pressure difference between the outside and inside of the tube.

$$
\mathrm{u}=\frac{\mathrm{dl}}{\mathrm{dt}}=\frac{\mathrm{r}^{2}}{8 \eta \mathrm{l}}\left[\frac{2 \gamma_{1} \cos \theta}{\mathrm{r}}+\Delta \mathrm{p}\right]
$$

If $\Delta \mathrm{p}$ is enough lower than outer pressure,

$$
\mathrm{l}^{2}=\left[\frac{2 \gamma \mathrm{l} \cos \theta}{\mathrm{r}}\right] \mathrm{t}
$$

Thus, $1^{2}$ is in proportion to the increasing time because the other factors $\left(r, \gamma_{1} \cos \theta, \eta\right)$ are constant. In the measurement, benzyl alcohol is used as the test liquid. The slope of Figure 7 is attributed to the surface energy that contained constants in equation (2). As shown in the Figure7, Oxide PE has highest surface energy.. Though the cross linked PEG PE shows second higher value, it should be chemically stable polymer layer in the air environment than that of oxide PE surfaces. PEG adsorbed PE

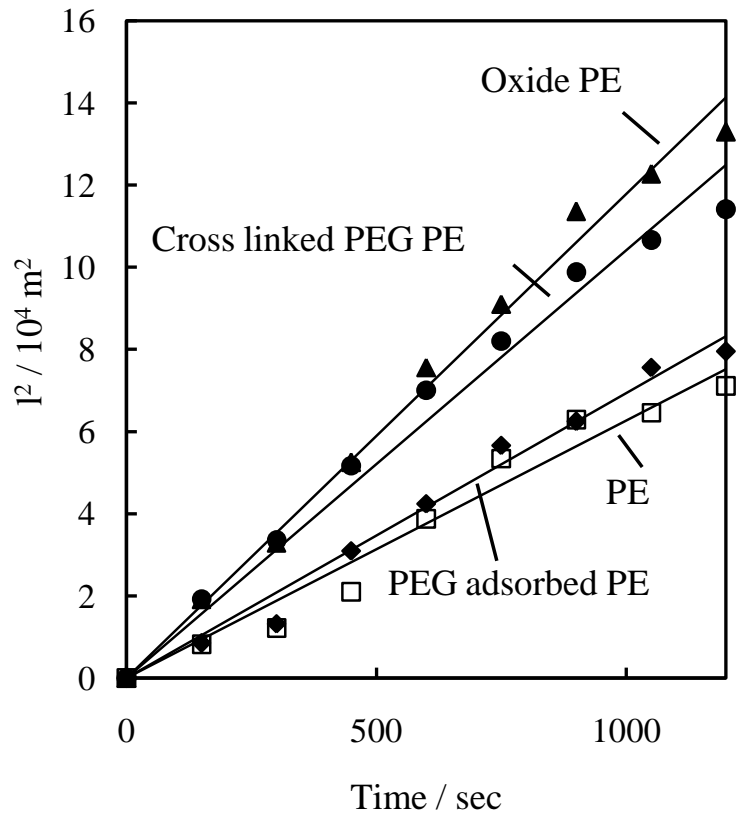

Fig. 7 Square of 1 as a function of the raising time

shows very close value as in untreated PE. Probably adsorbed raw PEG molecule was dissolved in the benzyl alcohol.

The solubility in the water of the cross linked PEG layer fabricated on the solid surface was tested by water resistance of the spin coated raw PEG layer on the glass surface. After APGD plasma surface treatment, the raw PEG surface was changed from soluble to insoluble in the water.

\section{Conclusion}

We succeed the water insoluble polyethylene glycol films as a core-shell of polymer powder by the combination techniques of adsorption of PEG on the powder surfaces and atmospheric glow discharge(APGD) irradiation to the surface. The cross linked PEG layer fabricated on the polymer powder show very hydrophilic nature on the surfaces.

\section{References}

[1] Alcantar N.A., Aydil E.S., Israelachvil J.N., J. Biomed.Mater.Res., 51(2000) 343

[2]Sheu M.S., Hoffman A.S., Feijen J. J.Adh.Sci.B, 4(1992)995

[3]R. Prat, T. Suwa, M. Kogoma and S. Okazaki, $J$. Adhession, 66, (1998) 163-182

[4]_M.Kogoma, S.Hashimoto and K.Tanaka, $J$. Photopolym. Sci. Tech.,19, (2006) 231-234 